\title{
Digestible Indispensable Amino Acid Score (DIAAS) better Estimates the Protein Value of Pistachios than Digestibility Corrected Amino Acid Score (PDCAAS)
}

\section{Abstract}

Pistachios (Pistacia Vera) are nutrient-dense foods with a healthy nutritional profile that contains fiber, unsaturated fatty acids, essential nutrients, phytochemicals and protein. However, the nutritional quality of the protein has not been characterized to date. Therefore, the objective of this study was to determine the Protein Digestibility Corrected Amino Acid Score (PDCAAS) and Digestible Indispensable Amino Acid Score (DIAAS) for raw and roasted American pistachio nuts in growing pigs. The values for apparent ileal digestibility (AID) of crude protein (CP) and the standard ileal digestibility (SID) where calculated for these proteins. The CP content was greater for raw pistachios (27.1\%) compared with roasted pistachios (25.1\%). The amino acid (AA) in greatest concentrations for both types of pistachio were arginine followed by leucine. The AA in the lowest concentration were tryptophan followed by methionine. The AID of $\mathbf{C P}$ was greater $(P<0.05)$ for raw pistachios than for roasted pistachios, and the AID of most indispensable AA (IAA), except arginine, isoleucine, and phenylalanine, was greater $(P<0.05)$ for raw pistachios. The SID of CP was greater $(P<0.05)$ for raw than for roasted pistachios, and the SID of all IAA, except phenylalanine, was greater $(P<0.05)$ for raw than for roasted pistachios. The PDCAAS value calculated for both types of pistachios was based on the requirement for children from 2 to 5 years old (FAO, 1991), whereas the DIAAS value was calculated based on the requirement for children 3 years and older, adolescents, and adults (FAO, 2013). Both raw and roasted pistachios had a PDCAAS value of 75. In contrast, the DIAAS was numerically greater for raw pistachios than for roasted pistachios with values of 86 and 83, respectively. For PDCAAS, the first limiting AA in both raw and roasted pistachios when compared with the AA requirements for children 2 to 5 years was threonine. For DIAAS, lysine was the first limiting AA in both raw and roasted pistachios when compared with the AA requirements for children 3 years and older, adolescents, and adults. Based on the DIAAS cut-off values describing protein quality (FAO, 2013), raw pistachios and roasted pistachios can both be considered a 'Good' quality protein if consumed by children older than 3 year, adolescents, and adults.

\section{Conflict of Interest}

Arianna Carughi is a consultant to American Pistachio Growers, a not for profit California Agricultural Board 